\title{
DIEZ AÑOS DE ESTUDIOS SOBRE EL TEMPLO MAYOR DE TENOCHTIT,LAN
}

(1978-1988)

\section{Introducción}

NELly GutIÉRREZ SOLANA

El 21 de febrero de 1978 se descubrió la escultura de la Coyolxauhqui y en marzo del mismo año se inició el Proyecto Templo Mayor bajo la atinada dirección del arqueólogo Eduardo Matos. Diez años han pasado y la importancia del proyecto y sus logros impresionantes han resultado en numerosos libros y artículos. Mucho se ha aprendido tanto de las excavaciones como de las reflexiones que en torno de los hallazgos han hecho los investigadores.

El propósito de esta guía es presentarle al estudioso un índice temático que le permita saber en qué publicaciones puede encontrar información sobre los diversos aspectos del Templo Mayor. Asimismo tendrá en sus manos la bibliografía más completa posible.

El arqueólogo Eduardo Matos y sus colaboradores han publicado los datos obtenidos en las excavaciones, lo mismo que el material fotográfico complementario, principalmente en el libro Templo Mayor: Excavaciones y Estudios, volumen 1. Esperamos con interés el volumen 2 del mismo y las tesis de los alumnos de la Escuela Nacional de Antropología que tratan de aspectos específicos del proyecto. Gracias a la eficiente labor arqueológica se han determinado las diversas etapas constructivas del Templo Mayor y poseemos amplia información sobre los objetos y las ofrendas desenterradas, así como una cronología tentativa de estas últimas (ver Broda, J., D. Carrasco y E. Matos, 1987). Para el estudio de las ofrendas, aparte del libro citado, son importantes el libro Templo Mayor: Excavaciones y Estudios, volumen 1, y el libro de Debra Nagao Mexica Buried Offerings: A Historical and Contextual Analysis (1985).

Las investigaciones sobre el simbolismo del Templo Mayor han sido particularmente enriquecedoras por las diferentes vías de acercamiento adoptadas por los autores. Muchos de ellos han advertido que sólo por medio de una colaboración interdisciplinaria se llegará a comprender el complejo simbolismo de dicho templo.

Eduardo Matos se acerca al significado del Templo Mayor a través de dos niveles: el de la estructura y el de la superestructura. La estructura 
de la sociedad mexica, cuya base era la agricultura y la guerra, se refleja en los dioses venerados en dicho templo: Tláloc y Huitzilopochtli. En cuanto a la superestructura, que engloba las creencias sobre el cosmos, considera que para los mexicas el Templo Mayor era el centro del universo, el punto en el cual el nivel celeste y el inframundo empezaban y donde se originaban las cuatro direcciones del mundo.

Johanna Broda opina que también dicho recinto sagrado refleja la cosmovisión de los mexicas y encuentra en las ofrendas alusiones a la tierra y al agua, así como al culto a las montañas y a las cuevas. La fauna marina hallada en las ofrendas sugiere que se quería evocar la presencia del mar en el Templo Mayor por las connotaciones de fertilidad que tenía el mismo.

Ciertos rituales llevados a cabo en el Templo Mayor permiten a autores como Cecilia Klein y Richard F. Townsend interpretar lo que significaba dicho recinto ceremonial para los mexicas. La primera trata de los autosacrificios practicados en él y su contexto político, mientras que el segundo habla de los ritos celebrados allí durante la coronación de los soberanos.

Las fuentes etnohistóricas arrojan luz sobre las funciones y actividades realizadas en el corazón del centro ceremonial, como lo demuestran los trabajos de Miguel León-Portilla. Dichas fuentes pueden utilizarse además para el fechamiento de las etapas constructivas del Templo Mayor. Las lápidas halladas en el sitio ayudan asimismo a establecer una cronología. Emily Umberger concentra su estudio en ellas y establece su relación con el calendario y con la historia, ya que dichas lápidas conmemoran el fin y el comienzo de un ciclo calendárico o la muerte de un gobernante y el ascenso del siguiente.

Frances F. Berdan se basa en su análisis de las ofrendas del Templo Mayor para comprender el papel del comercio y del tributo en la economía mexica. Por su parte, David Carrasco explica varios aspectos políticos y sociales por el tipo de relaciones existentes entre el centro, es decir, Tenochtitlan, y la periferia donde estaban situados los pueblos tributarios. Para él es particularmente importante la expansión de arquetipos religiosos para el control de las comunidades periféricas.

Los estudios iconográficos han permitido identificar a las deidades representadas en las esculturas y en las vasijas. Como ejemplo tenemos el penetrante análisis de Alfredo López Austin sobre la imagen del Dios del Fuego con máscara, en el cual emplea la iconografía para descubrir códigos antiguos referentes a sistemas de creencias cosmogónicas. Otros tra- 
tados iconográficos son: los de Carmen Aguilera y Henry Nicholson acerca de la Coyolxauhqui, el de Eduardo Matos enfocado a los dioses de las urnas funerarias de barro y el de Alfredo López Austin dedicado a la llamada Piedra Verde. Varios investigadores han publicado sus opiniones sobre la identificación de las esculturas masculinas sedentes con dos protuberancias como tocado, entre ellos: Henry B. Nicholson, Eduardo Matos, Debra Nagao, Johanna Broda y Esther Pasztory.

\section{Secciones del Indice Temático.}

I. Proyecto interdisciplinario del Templo Mayor: fases del proyecto, situación del Templo Mayor, excavaciones, estratigrafía, cortes transversales y localización de la Coyolxatihqui.

II. Arquitectura: planos y plantas de los edificios, orientación, etapas constructivas, técnicas y construcciones.

III. Escultura, pintura y cerámica: dentro de cada uno de estos incisos se tratan obras importantes en particular; además hay un inciso sobre la conservación y restauración de los monumentos.

IV. Objetos ceremoniales: braseros, cajas de piedra y la piedra de sacrificio.

V. Ofrendas con dos incisos al final: figuras de tipo mixteco y objetos procedentes del estado de Guerrero y del estilo Mezcala.

VI. Glifos en lápidas desenterradas en el sitio y los que aparecen en figurillas y máscaras.

VII. Simbolismo del Templo Mayor.

VIII. Economía, comercio y tributo.

IX. Historiografia de los hallazgos realizados en el centro de Tenochtitlan.

En este índice no incluyo investigaciones sobre la época colonial ni tampoco las fuentes etnohistóricas que se refieren al Templo Mayor. Mi punto de partida es el año de 1978; las publicaciones anteriores han sido cubiertas detalladamente en el artículo de Elizabeth Boone titulado “Templo Mayor Research, 1521-1978", publicado en 1987.

Para terminar esta introducción quiero agregar unas cuantas notas explicativas: a) en el índice temático las publicaciones siguen un orden cronológico, en lugar de un orden alfabético por autores, para poder así seguir el desarrollo de los estudios y de las interpretaciones; b) el lector interesado en un tema deberá buscarlo en dicho índice, donde encontrará 
el autor, la fecha de la publicación y las páginas que tratan del tema, y después hallará en la bibliografía los demás datos pertinentes a la publicación; c) si no está incluida la páginación en los datos del índice es que todo el artículo o todo el libro se refiere al tema, y d) cuando varias publicaciones del mismo autor van seguido en el índice se omite el nombre del autor y se pone únicamente el año y la paginación. En el caso de algunas ofrendas no hay datos publicados sobre su contenido y por eso no aparecen en el índice.

Agradezco al arqueólogo Eduardo Matos y al doctor Hasso von Winning su ayuda en la realización de este trabajo.

Indice Temático (referencias principales)

Abreviaturas usadas:

ENAH Escuela Nacional de Antropología e Historia.

UNAM Universidad Nacional Autónoma de México.

INAH Instituto Nacional de Antropología e Historia.

SEP Secretaría de Educación Pública.

BCM Broda, J., D. Carrasco y E. Matos, 1987, The Great Temple of Tenochtitlan.

\section{Proyecto del Templo Mayor.}

Fases del proyecto, Matos, 1978: 3-17; 1979a: 30-33; 1979b: 18-26; 1981b: 117-123; 1982b: 11, 12; 1984a: 42-47; 1986b: 249-252; 1986c: 94-101; Broda, Carrasco y Matos, 1987: 23-25.

Situación del Templo Mayor, Matos, 1980b: 771; 1981b: 140; 1984a: 11; Aveni et al., 1988.

Excavaciones, García Cook y Ârana, 1978; Contreras y Luna, 1982; Gutiérrez, 1982; Hernández, 1982a; Matos, 1982b; 1982d; Wagner, 1982.

Estratigrafia, García Cook y Arana, 1978; Reyes Cortés, 1978; Matos, 1982b.

Cortes transversales y localización de la Coyolxauhqui, García Cook, 1978; García Cook y Arana, 1978.

\section{Arquitectura.}

Planos, Matos, 1980b: 768, 769; 1981b: 136-138, 141-143; 1981c; 1982c; 1984a: 4, 5; 1984c; Umberger, 1987, fig. 1; Villalobos, 1985. Plantas del Templo Mayor con las ofrendas y hallazgos, Matos, 1980a; 
1981b: 141; Bonifaz, 1981: 178, 179; Matos, 1982c; Nagao, 1985 a. Orientación, Aveni et al., 1988; Aveni y Hartung, s. f. Etapas constructivas, León Portilla, 1981; Matos, 1981b: 142, 143, 162, $168,169,256,257,259,268$; 1981c; Nicholson, 1982; Matos, 1984a: 119; 1984c; Baquedano, 1987: 92-98; Broda, Carrasco y Matos, 1987: 67, 68; Nicholson, 1987; Townsend, 1987; Umberger, 1987; Matos, 1988: 64-83.

Arquitectura en general, Molina, 1980: 753-764; Matos, 1981c; 1982b; 1984a; Molina, 1987.

Técnicas constructivas, Molina, 1980; Matos, 1981b: 256, 258, 259, 267; Molina, 1987: 103-105.

Plataforma general, Matos, 1981c: 23, 29; 1982d; 1984b: 16-18, foto 2. Basamento del Templo Mayor, Matos, 1982d; Molina, 1987: 99-101.

Coatepantli, Matos, 1981b: 267; 1982b; Molina, 1987: 98, 100.

Adoratorio de Tláloc, Matos, 1981b: 153, 154, 158, 159, 160; 1981c: 19; 1982d: 44; 1984a: 86-90; 1988: 66.

Adoratorio de Tláloc, Matos, 1981b: 153, 154, 158, 159, 160; 1981c: 19-23; 1982a: 114, 115; 1982d: 41, 44, 47; 1984a: 90-94, foto: 91; 1988: 66. Restos de madera, Matos, 1981b: 160; Gómez Mont, 1982: 233-240; Matos, 1984a: 94.

Adoratorio A, Matos, 1981c: 41; 1982d: 63-65; 1984a: 101; 1984b: 18; 1988: 78.

Adoratorio B (altar-tzompantli), Matos, 1981b: 275; 1981c: 41; 1982d: 63, 64; 1984a: 101, 102, 114, foto: 97; 1984b: 18, 19, 21, foto 3; 1988: 78.

Adoratorio C, Matos, 1981b: 276, 277; 1981c: 41; 1982d: 65, 66; 1984a: 102, 103, 114, foto: 100; 1984b: 19; Molina, 1987: 99; Matos, 1988: 79.

Adoratorio D, Matos, 1984a: 114; 1984b: 19.

Templo Rojo, Matos, 1981b: 258-265, 267; 1982b: 42, 54, 60; Molina, 1987: 99; Townsend, 1987, fig. 10.

Edificio Norte, Matos, 1981b: 267, 278; 1982d: 61.

Altar con ranas, Matos, 1981b: 180, 181, 190; 1981c: 29; 1982d: 56; 1984a: 71, foto: 72; 1988: 73, foto 43 .

Basamento de las Águilas, Matos, 1981b: 267, 279-283; 1981c: 41; Gutiérrez, 1982: 105; Matos, 1982d: 55, 56, 61, 62; 1984a: 103, fotos: 108, 109, 115; 1984b: 19; Broda, 1987, figs. 30, 31; Molina, 1987: 102. Recinto de los Caballeros Águila, Matos, 1984a: 106-114; 1984b: 1921, fotos: 4-15; Broda, 1987: 239; Klein, 1987: 304-314, figs. 7, 8; 
Molina, 1987: 102, 103; Matos, 1988: 82, 83.

Patio posterior, (patio de los altares), Matos, 1981c: 41; Contreras y Luna, 1982; Matos, 1982d: 60; 1984a: 115, 116, foto: 116; 1984b: 20 , foto: 16 .

III. Escultura, pintura y cerámica.

Banquetas con procesiones de guerreros, Matos, 1981b: 256, 1982d: 55, 62, 70; Pasztory, 1983: 144, 145, foto: 145; Matos, 1984a: 107, 110, 111, 114, fotos: 101, 103; 1984b: 19-21, fotos 4-8; Broda, 1987, fig.: 240; Klein, 1987: 298-314, fig. 8; Molina, 1987: 102, 103; Townsend, 1987, fig. 11.

Caballeros Aguila en barro, Nicholson y Quiñones, 1983: 84, 85; Matos, 1984a: 107,111 , fotos: 104,105 ; 1984b: 19-21, fotos 13-15; Klein, 1987, fig. 13; Matos, 1988: 101, 102.

Cabeza de Xipe, Matos, 1980a: 35, 77; Bonifaz, 1981: 80, 81.

Caracoles marinos esculpidos en piedra, Matos, 1980a: 36, 37, 73; 1980b: 775; Bonifaz, 1981: 96, 97; Matos, 1981b: 266; Contreras y Luna, 1982: 92; Luna, 1982, 241-244; Matos, 1982d: 39, 61; Nicholson y Quiñones, 1983: 110, 111; Matos, 1984a: 116, 117, foto: 110 , 111; Catảlogo, 1986, fig. 285; Matos, 1988: 99.

Coyolxauhqui, Aguilera, 1978a; 1978b; Blanco, 1978; García Cook, 1978; García Cook y Arana, 1978; León Portilla, 1978: 48-51; Peña Gómez, 1978: 39-51; Aguilera, 1979; 1980a; 1980b; Matos y Ehrenberg, 1980; Bonifaz, 1981: 16, 52; Matos, 1981b: 186, 201; Franco, 1982; Matos, 1982d: 23; Riese, 1982; Graulich, 1983: 99; Pasztory, 1983: 154, 155, fotos: 154, 155; Matos, 1984a: 23, 24, 71, 72, 73, fotos: 68, 69; Nicholson, 1985; Winning, 1985; Broda, Carrasco y Matos, 1987; 104, 105.

Coyolxauhqui II, García Cook, 1978: 27; García Cook y Arana, 1978: 55-57, fig. 48; Matos, 1981b: 201.

Chacmool, Bonifaz, 1981: 116, 117; Matos, 1981b: 148-153; Franco, 1982; Matos, 1982d: 41, 42; Pasztory, 1983: 144, foto: 180; Matos, 1984a: 90, foto: 88; Broda, 1987: 239; Matos, 1988: 66.

Dios del Fuego con máscara, Matos, 1982d: 70; Nicholson y Quiñones, 1983: 34, 35; Pasztory, 1983: 175, foto: 176; López Austin, 1987; Matos, 1988: 99, 101; fotos: $84,85$.

Esculturas antropomorfas que descansaban en las escalinatas, Matos, 1980a: 63, 78, 83; 1981b: 132, 133, 162, 167; 1981c: 23; Hernández Pons, 1982b; Matos, 1982d: 27, 38, 39; 1984a: 79-81, fotos: 78, 79; 
Catálogo, 1986, fig. 302; Matos, 1988; 73, láms.: 138, 139. Escultura en piedra verde, García Cook, 1978: 27; García Cook y Arana, 1978: 58-66, 69; López Austin, 1979; Matos, 1980a: 55, 75; 1981b: 191; Franco, 1982; Pasztory, 1983: 155-157, foto: 156; Nagao, 1985a: 71, 72; Broda, Carrasco y Matos, 1987: 104; Matos, 1988: 96.

Esculturas masculinas sedentes con dos protuberancias como tocado, García Cook y Arana, 1978: 39, 41; Matos, 1980a: 59, 62, 74; Bonifaz, 1981: 127, 129, figs. 91, 314, 316; Matos, 1981b: 206-208, 210, 213, 219, 256; Ahuja, 1982a: 203; Contreras y Luna, 1982: 86; Matos, 1982d: 49; Wagner, 1982; Pasztory, 1983: 223-225, fotos: 217, 224; Nagao, 1985a: 64-70, 85-87, 99, 101; 1985b; Broda, 1987: 232-234; Broda, Carrasco y Matos, 1987: 37-95; Matos, 1988: 92, 94; Nagao, s. f.; Nicholson y Quiñones, 1983: 88, 89.

Escultura sedente del Dios del Pulque, Nicholson y Quiñones, 1983: 90, 91.

Figuras esqueléticas en barro, Matos, 1984a: 107, 111, foto: 103, 1984b: 20, 21, fotos: 6, 11, 12; Klein, 1987: 307, fig. 10.

Lápida con serpientes en relieve que forma parte del escalón de la plataforma, Matos, 1981b: 187; 1981c: 29; 1982d: 56; 1988: 73.

Máscara mexica en alabastro, Matos, 1981b: 255, 258.

Máscara olmeca, Matos, 1979c; 1980a: 31, 73; Bonifaz, 1981: 22, 23; Matos, 1981b: 190, 192, 196, 197, 258; 1982d: 35, 185-190; Wagner, 1982: 138; Matos, 1988: 113, 114.

Máscaras tipo teotihuacano, Matos, 1980a: 30, 73; Bonifaz, 1981: 6870; Matos, 1981b: 190, 192, 196, 198, 199, 254, 258; 1982d: 60; Wagner, 1982: 138, 139; Catálogo, 1983, figs. 324, 339; Matos, 1984a, foto: 56, 57; Nagao, 1985a: 74; Matos, 1988: 114.

Mayahuel, ver Escultura en piedra verde.

Pectoral en piedra verde con una deidad (Coyolxauhqui?), García Cook y Arana, 1976: 64; Matos, 1981b: 91.

Portaestandartes, ver Esculturas antropomorfas que descansaban en las escalinatas.

Ranas, Contreras y Luna, 1982: 77, 84, 92. Ver también Altar con ranas. Relieve de dos Tlálocs sobrepuestos, León Portilla, 1978, foto: 57; Matos, 1982d: 19; 1984d: 146, 147; Catálogo, 1986, fig. 341; Broda, 1987, fig. 33.

Relieve de flor con cuatro pétalos, Matos, 1984a: 107, fotos: 101, 106; 1984b: 20, fotos 6, 7 .

Serpientes, cabezas de, Bonifaz, 1981: 104-109; Matos, 1981b: 168, 169, 
175-179, 182-185, 188-190, 214; 1981c: 29; Contreras y Luna, 1982: 80, 81, 100; Matos, 1982d: 27, 50, 51, 54, 56, 60; Graulich, 1983; Matos, 1984a: 70-72, fotos: 63, 67, 71; Gutiérrez Solana, 1987: 124, 126, 128, fotos: 39, 86, 87, 120, 134; Molina, 1987: 101, 102.

Serpientes de cuerpos ondulantes, Matos, 1981b: 169-171, 190; 1981c: 29; Matos, 1982d: 56; Matos, 1984a, foto: 95; Gutiérrez Solana, 1987: 126, 128, fotos: 117, 119; Matos, 1988: 73.

Serpiente enrollada policroma, Matos, 1980a: 76; Matos, 1982d: 49; Catálogo, 1986, fig. 303.

Tláloc, ver también Escultura con dos Tlálocs sobrepuestos y Vasijas con Tlálocs. Matos, 1980a: 31, 58, 74, 76, 78; Bonifaz, 1981, fig. 59; Matos, 1981b: 194, 195, 204, 205, 214, 216, 241-243; Ahuja, 1982: 203; Contreras y Luna, 1982: 74, 75, 77, 81, 84, 85, 92; González, 1982; Matos, 1982d: 32, 33, 40, 49, 52, 53; Wagner, 1982; Heyden, 1984: Matos, 1984a, foto: 102; 1984b: 20, fotos 9, 10; Nagao, 1985a: 71; Broda, 1987: 214; González, 1987: 153, fig. 6.

Tlaltecuhtli, Matos, 1981b: 268; 1982d: 70; Nicholson y Quiñones, 1983: 60, 61; Broda, 1987: 240-245, fig. 32; BCM, 1987: 102-105; Matos, 1988, lám. 128.

Vasijas con Tláloc, (de piedra y de barro), García Cook y Arana, 1978: 39, 42; Matos, 1980a: 27, 29, 73, 74; Bonifaz, 1981, figs. 54, 86, 90; Matos, 1981b: 168, 202, 203, 206, 208, 213, 242, 252, 253, 256, 258; Contreras y Luna, 1982: 83, 86; Hasbach, 1982a; Matos, 1982d: 45, 55, 62, 70; Wagner, 1982; Nicholson y Quiñones, 1983: 10, 92, 93; Heyden, 1984: 23-32, fig. 1; Matos, 1984a, fotos: 49-51; Nagao, 1985a: 71, 85; Catálogo, 1986, figs. 276, 279; González, 1987: 154, fig. 7; Heyden, 1987: 110-113; Matos, 1988: 94.

Vaso con Mictlantecuhtli, Matos, 1980a: 39, 77; Bonifaz, 1981: 82-84; Catálogo, 1986, fig. 298; Matos, 1988: 96.

Pinitura.

Pintura mural, Contreras y Luna, 1982: 96; Santaella, 1982.

Pintura mural del adoratorio de Huitzilopochtli, Matos, 1982d: 43; Santaella, 1982; Matos, 1988: 66.

Pintura mural del adoratorio de Tláloc, Matos, 1981b: 153-158, 159161; 1981c: 23; 1982a: 114; 1982d: 41; Santaella, 1982; Matos, 1984a: 90-93, foto: 91; Matos, 1988: 66.

Pintura mural en adoratorio, Matos, 1982d: 40; Santaella, 1982.

Pintura mural Templo Rojo y Adoratorio C, Matos, 1981b: 258, 259, 267, 277; Matos, 1982d: 54; Santaella, 1982. 


\section{Cerámica.}

Cerámica del Templo Mayor, Matos, 1981b: 155, 162, 206, 242, 244, 245, 247, 250, 251; Ahuja, 1982b: 245-252; Catálogo, 1986, figs. 283, 307, 310; Heyden, 1987.

Urnas funerarias, Matos, 1980a: 26, 73, 75; Bonifaz, 1981: 85-90; Matos, 1981b: 157, 160, 205, 224; Mercado, 1982, fotos: 349-356; Matos, 1982d: 32, 33, 45; Wagner, 1982; Matos, 1983; Nicholson y Quiñones, 1983: 94-97; Matos, 1984a: 90, fotos: 42, 43; Catálogo, 1986, fig. 297; Nicholson, 1987: 475-477; Umberger, 1987: 428-436, figs. 9, 10; Matos, 1988: 106, 110, 111.

Conservación y restauración de las obras, Franco, 1982; Hasbach, 1982a; Hasbach, 1982b; Mercado 1982; Santaella, 1982; Franco, 1986; Franco, s. f.

IV. Objetos ceremoniales.

Braseros, Matos, 1981b: 168, 169, 172-175, 177; 1981c: 23, 29; Contreras y Luna, 1982: 80, 81; Matos, 1982d: 22, 45, 54; 1984a: 78, fotos: 73-76, 102; 1984b: 20, fotos: 9, 10; Broda, 1987, fig. 31; Heyden, 1987: 118, 119; Matos, 1988: 73, 97.

Cajas de piedra, Matos, 1980a: 42, 43, 78; González, 1982; Matos, 1982d: 41, 42, 48, 53, 66, 68, 70; Wagner, 1982; González, 1987: 154, fig. 8.

Piedra de sacrificios, Bonifaz, 1981: 118, 119; Matos, 1981b: 138, 148, 149, 152; 1982d: 43; Matos, 1984a: 87-89; Matos, 1988: 65.

V. Ofrendas.

Ofrendas en general y su significado, Matos, 1981b: 141, 190, 191, 256; 1981c: 29, 37; 1982a: 117, 118; Wagner, 1982; Matos, 1984a: 47-56; 1984d: 149-161; Nagao, 1985a; Baquedano, 1987: 98-102; Broda, 1987; BCM, 1987; 36-47, 84, 87, 88; Matos, 1988: 92-121; Nagao, s. f.

Cámara I, Blanco Padilla, 1978: 36, 37; García Cook, 1978; García Cook y Arana, 1978: 58-65; Matos, 1980a: 75; 1981b: 191; Nagao, 1985a: 116; BCM, 1987: 42.

Cámara II, Matos, 1980a: 73, 75, 76, 78; Bonifaz, 1981: 46, 51, 59, 77, 80, 81, 85; Matos, 1981b: 205, 216-219, 223, 228, 229; Ahuja, 1982a: 191-212; Matos, 1982d: 36-40; 1984a, fotos: 80, 81; Nagao, 1985a: 116; Catálogo, 1986, figs. 286, 319, 339, 340; Broda, 1987, figs. 17, 18, 26; BCM, 1987: 42; González, 1987: 153.

Cámara III, Matos, 1981b: 242, 256; 1982d: 60; 1984a, foto: 48; Na- 
gao, 1985a: 117; Catálogo, 1986, figs. 296, 307, 331; BCM, 1987: 42; González, 1987, figs. 4, 154.

Ofrenda 1, Blanco Padilla, 1978: 32, 33, 35; García Cook, 1978; García Cook y Arana, 1978: 37-49; Peña Gómez, 1978; Bonifaz, 1981, figs. 27, 48; Matos, 1981b: 191; Nagao, 1985a: 114; BCM, 1987: 42.

Ofrenda 2, García Cook, 1978; García Cook y Arana, 1978: 50; Nagao, 1985a: 115; BCM, 1987: 42.

Ofrenda 3, Blanco Padilla, 1978: 36; García Cook, 1978; García Cook y Arana, 1978: 51-53; Bonifaz, 1981, fig. 45; Nagao, 1985a: 115; BCM, 1987: 43; Umberger, 1987: 428, 429.

Ofrenda 4, García Cook, 1978; García Cook y Arana, 1978: 54-57; Nagao, 1985a: 116; BCM, 1987: 41.

Ofrenda 5, Blanco Padilla, 1978: 36, 37; García Cook, 1978; García Cook y Arana, 1978: 58-65; Nagao, 1985a: 116; BCM, 1987: 41.

Ofrenda 6, Matos, 1980a: 74-76; Bonifaz, 1981, figs. 23, 49, 53, 57, 60, 69, 74, 75, 78, 82, 87, 91; Matos, 1981b: 12, 13, 229, 230, 231; 1982d: 23; Wagner, 1982; Nicholson y Quiñones, 1983: 90, 91; Nagao, 1985a: 117; Catálogo, 1986, figs. 277, 278, 292, 294, 300, 311, 313, 314; BCM, 1987: 43.

Ofrenda 7, Bonifaz, 1981, fig. 61; Matos, 1981b: 208, 213-215, 256; Álvarez, 1982: 161-172; Álvarez et al., 1982: 173-184; Díaz-Pardo, 1982: 151-160; Matos, 1982d: 25, 28; Polaco, 1982: 143-150; Wagner, 1982; Matos, 1984a, foto: 60; Nagao, 1985a: 118; BCM, 1987: 36, 43. Ofrenda 8, Matos, 1981b: 168; 1982d: 26, 29-31; Wagner, 1982; Nagao, 1985a: 119; BCM, 1987: 40.

Ofrenda 9, Bonifaz, 1981, fig. 84; Matos, 1982d: 31, 32; Wagner, 1982; Nagao, 1985a: 119; BCM, 1987: 43.

Ofrenda 10, ver también urnas funerarias, Matos, 1980a: 73; 1981b: 224; 1982d: 32; Wagner, 1982; Nicholson y Quiñones, 1983: 94, 95; Nagao, 1985a: 120; BCM, 1987: 43; Nicholson, 1987: 475-477; Umberger, 1987: 428-436.

Ofrenda 11, Matos, 1980a: 74-77; Bonifaz, 1981, figs. 50, 58, 63, 7072, 76, 89; Matos, 1981b: 214, 248; 1982d: 34; Wagner, 1982; Matos, 1984a, foto: 61; Nagao, 1985a: 120; Catálogo, 1986, figs. 284, 289, 293, 305; BCM, 1987: 37, 43.

Ofrenda 12, Contreras y Luna, 1982: 82; Wagner, 1982; Nagao, 1985a: 121; BCM, 1987: 43.

Ofrenda 13, Matos, 1981b: 214, 225, 226; 1982d: 36, 37; Nagao, 1985a: 121; Catálogo, 1986, fig. 322; BCM, 1987: 43. 
Ofrenda 14, ver también urnas funerarias, Matos, 1980a: 73, 77; 1981b: 224; 1982d: 33; Wagner, 1982; Nicholson y Quiñones, 1983: 96, 97; Nagao, 1985a: 121; BCM, 1987: 43; Nicholson, 1987: 475-477; Umberger, 1987: 428-436, fig. 9 .

Ofrenda 15, Contreras y Luna, 1982: 82, 85; Matos, 1982d: 34; Wagner, 1982; Nagao, 1985a: 122; BCM, 1987; 44.

Ofrendas 16 y 16- $A$, Contreras y Luna, 1982: 83, 86; Matos, 1982d: 34; Wagner, 1982; Nagao, 1985a: 122; Broda, 1987, fig. 28.

Ofrenda 17, Matos, 1980a: 74, 76; Bonifaz, 1981, fig. 86; Matos, 1981b: 207, 253, 258; Contreras y Luna, 1982: 82, 86; Matos, 1982d: 34; Wagner, 1982; Nagao, 1985a: 122; Catálogo, 1986, figs. 299, 316; BCM, 1987: $37,44$.

Ofrenda 18, Matos, 1981b: 205, 214; 1982d: 34; Wagner, 1982; Nagao, 1985a: 123; Broda, 1987, fig. 19; BCM, 1987: 44.

Ofrencia 19, Matos, 1981b: 204, 205, 214; 1982d: 34; Wagner, 1982; Nagao, 1985a: 124; Broda, 1987, figs. 20, 21; BCM, 1987: 44.

Ofrenda 20, ver también máscara olmeca, Matos, 1980a: 73, 77; Bonifaz, 1981, figs. 22, 24, 29, 44, 55, 67; Matos, 1981b: 168, 190, 192m 199, 222, 252, 258; Contreras y Luna, 1982: 82; Matos, 1982d: 34, 35, 185-190; Wagner, 1982; Matos, 1979c; Nagao, 1985a: 124; Catálogo, 1986, figs. 288, 298, 320, 321, 324; BCM, 1987: 44.

Ofrenda 21, ver también vasijas con Tlálocs, Matos, 1980a: 73; Bonifaz, 1981, fig. 54; Matos, 1982d: 34, 38, 41; Nicholson y Quiñones, 1983: 92, 93; Heyden, 1984: 23-32, fig. 1; Nagao, 1985a: 125; Catálogo, 1986, fig. 276; BCM, 1987: 40; Heyden, 1987: 110-113.

Ofrenda 22, Matos, 1982d: 34, 40; Nagao, 1985a: 125; BCM, 1987: 44.

Ofrenda 23, Matos, 1981b: 206; 1982d: 37, 40, 41; Nagao, 1985a: 125; Catálogo, 1986, fig. 301; BCM, 1987: 44.

Ofrenda 24, Matos, 1980a: 77; Bonifaz, 1981, fig. 39; Matos, 1981b: 206; 1982d: 38-40; Nagao, 1985a: 126; Catálogo, 1986, figs. 282, 306; BCM, 1987: 45 .

Ofrenda 25, Matos, 1982d: 39; Nagao, 1985a: 126; BCM, 1987: 40. Ofrenda 26, Nagao, 1985a: 126; BCM, 1987: 41.

Ofrenda 27, Contreras y Luna, 1982: 92; Nagao, 1985a: 126.

Ofrenda 28, Nagao, 1985a: 126; BCM, 1987: 41.

Ofrenda 29, Matos, 1982d: 41, 42; Nagao, 1985a: 126; BCM, 1987: 41. Ofrenda 30, Matos, 1981b: 206; 1982d: 42, 45; Nagao, 1985a: 127; Broda, 1987, fig. 11; BCM, 1987: 41. 
Ofrenda 31, Matos, 1980a: 73; Bonifaz, 1981, fig. 90; Matos, 1981b: 206; 1982d: 45; Nagao, 1985a: 127; BCM, 1987: 41; González, 1987: 154, fig. 1.

Ofrenda 32, Matos, 1982d: 45; Nagao, 1985a: 127; BCM, 1987: 41. Ofrenda 33, Matos, 1982d: 45; Nagao, 1985a: 127; BCM, 1987: 40. Ofrenda 34, Matos, 1980a: 75; Bonifaz, 1981, figs. 40, 83; Matos, 1981b: 160; 1982d: 45; Nagao, 1985a: 127; BCM, 1987: 40.

Ofrenda 35, Matos, 1981b: 160; Nagao, 1985a: 128; BCM, 1987: 41. Ofrenda 36, Matos, 1982d: 45; Nagao, 1985a: 128; BCM, 1987: 41. Ofrenda 37, Matos, 1981b: 160; 1982d: 47; Nagao, 1985a: 128; BCM, 1987: 40.

Ofrenda 38, Matos, 1981b: 160; 1982d: 47; Nagao, 1985a: 128; BCM, 1987: 40.

Ofrenda 39, Matos, 1981b: 157; 1982d: 47; Nagao, 1985a: 128, Catálogo, 1986, figs. 297, 325-327, 334, 336-338; BCM, 1987: 40.

Ofrenda 40, Matos, 1982d: 47; Nagao, 1985a: 129; BCM, 1987: 40. Ofrenda 41, Matos, 1980a: 78-82; Bonifaz, 1981, figs. 34-36, 47, 65, 73; Matos, 1981b: 200, 201, 206; González, 1982: 213-220; Matos, 1982d: 47, 48; Nagao, 1985a: 129; Broda, 1987, figs. 7, 8; González, 1987: 154; BCM, 1987: 45.

Ofrenda 42, Nagao, 1985a: 129; BCM, 1987: 40.

Ofrenda 43, Matos, 1982d: 48; Nagao, 1985a: 130; BCM, 1987: 41.

Ofrenda 44, Matos, 1980a: 77; Bonifaz, 1981, fig. 56; Matos, 1981b: 155, 160; 1982d: 48; Nagao, 1985a: 130; BCM, 1987: 40.

Ofrenda 45, Matos, 1982d: 48: Nagao, 1985a: 130; BCM, 1987: 40. Ofrenda 46, Matos, 1982d: 48; Nagao, 1985a: 130; BCM, 1987: 41. Ofrenda 47, Matos, 1982d: 48; Nagao, 1985a: 130; BCM, 1987: 41. Ofrenda 48, Bonifaz, 1981, fig. 21; Matos, 1981b: 219, 241-243, 246, 247; 1982d: 51-53, 55; Nagao 1985a: 130; BCM, 1987: 45; Román, 1987; Román, s. f.

Ofrenda 49, Matos, 1982d: 49; Nagao, 1985a: 131; BCM, 1987: 47.

Ofrenda 50, Matos, 1982d: 49; Nagao, 1985a: 131.

Ofrenda 51, Matos, 1982d: 49; Nagao, 1985a: 131; BCM, 1987; 47.

Ofrenda 52, Matos, 1981b: 268, 270, 271; 1982d: 49; Nagao, 1985a: 131; Broda, 1987, fig. 34.

Ofrenda 53, Matos, 1982d: 52; Nagao, 1985a: 131; BCM, 1987: 45. Ofrenda 54, Matos, 1981b: 248, 251; 1982d: 52, 53, 55; Nagao, 1985a: 132; BCM, 1987: 46.

Ofrenda 55, Matos, 1982d: 52, 53; Nagao, 1985a: 132; BCM, 1987: 41. 
Ofrenda 56, ver también vasijas con Tlálocs, Matos, 1981b: 202, 203; 1982d: 52, 53, 55; Nicholson y Quiñones, 1983: 93; Heyden, 1984: 23-32; Nagao, 1985a: 132; BCM, 1987: 41; Heyden, 1987: 110-113. Ofrenda 57, Matos, 1982d: 54; Nagao, 1985a: 132; Catálogo, 1986, figs. 290, 291, 295.

Ofrenda 58, Matos, 1981b: 232-235, 237; 1982d: 53, 55; Nagao, 1985a: 133; Catálogo, 1986, figs. 280, 310; BCM, 1987: 45.

Ofrenda 59, Matos, 1982d: 55.

Ofrenda 60, Matos, 1982d: 55, 60; Nagao, 1985a: 133; Catálogo, 1986, fig. 279; BCM, 1987: 45.

Ofrenda 61, Matos, 1981b: 206, 208-213; 1982d: 55; Nagao, 1985a: 133; Catálogo, 1986, fig. 312; BCM, 1987: 36, 45.

Ofrenda 62, Matos, 1982d: 60; Nagao, 1985a: 133; BCM, 1987: 45.

Ofrenda 63, Nagao, 1985a: 133; BCM, 1987: 45.

Ofrenda 64, Nagao, 1985a: 133; BCM, 1987: 47.

Ofrenda 65, Nagao, 1985a: 134.

Ofrenda 67, Nagao, 1985a: 134; BCM, 1987: 46.

Ofrenda 69, Nagao, 1985a: 134; BCM, 1987: 46.

Ofrenda 70, Nagao, 1985a: 134; BCM, 1987: 47.

Ofrenda 75, Nagao, 1985a: 134; BCM, 1987: 46.

Ofrenda 76, Nagao, 1985a: 134; BCM, 1987: 46.

Ofrenda 77, Nagao, 1985a: 135; BCM, 1987: 46.

Ofrenda 78, Matos, 1981b: 264, 265, 267; 1982d: 60; Nagao, 1985a: 135; Catálogo, 1986, fig. 304.

Ofrenda 79, Nagao, 1985a: 135; BCM, 1987: 47.

Ofrenda 80, Nagao, 1985a: 135; BCM, 1987: 46.

Ofrenda 81, Nagao, 1985a: 135; BCM, 1987: 47.

Ofrenda 82, ver también Máscaras de tipo teotihuacano, Matos, 1981b: 254-256; 1982d: 60; Nagao, 1985a: 135; BCM, 1987: 46.

Ofrenda 83, Nagao, 1985a: 136; BCM, 1987: 46.

Ofrenda 84, Matos, 1982d: 70; Nagao, 1985a: 136; BCM, 1987: 47.

Ofrenda 85, Catálogo, 1986, figs. 283, 308; González, 1987: 153; BCM, 1987: 41.

Ofrenda 86, BCM, 1987: 47.

Ofrenda 87, Catálogo, 1986, fig. 328; González, 1987: 153; BCM, 1987: 47.

Ofrenda 89, Catálogo, 1986, figs. 332, 333.

Ofrenda A, Gutiérrez, 1982: 105, 106; Matos, 1982d: 56, 66. 
Ofrenda $B$, Gutiérrez, 1982: 106; Matos, 1982d: 56, 66; Nagao, 1985a: 136.

Ofrenda C, Matos, 1982d: 66; Nagao, 1985a: 136.

Ofrenda D, Matos, 1982d: 68; Nagao, 1985a: 137. Ofrenda E, Matos, 1982d: 68; Nagao, 1985a: 137. Ofrenda F, Matos, 1982d: 68; Nagao, 1985a: 137. Ofrenda G, Matos, 1982d: 68; Nagao, 1985a: 137.

Ofrenda $H$, Matos, 1982d: 68; 1984a, fotos: 46, 47; Nagao, 1985a: 137; Catálogo, 1986, figs. 287, 317, 330.

Ofrenda I, Matos, 1982d: 68; Nagao, 1985a: 137. Ofrenda J, Matos, 1982d: 68; Nagao, 1985a: 138. Ofrenda K, Matos, 1982d: 68; Nagao, 1985a: 138. Ofrenda L, Matos, 1982d: 70; Nagao, 1985a: 138. Ofrenda $M$, Matos, 1982d: 70; Nagao, 1985a: 138. Ofrenda N, Matos, 1982d: 70; Nagao, 1985a: 138.

Figuras de tipo mixteco, Matos, 1980a; Ẃagner, 1982; Catálogo, 1986, figs. $277,278,284$.

Figuras y máscaras procedentes del estado de Guerrero y del estilo Mezcala, García Cook y Arana, 1978: 58, 62-64; Matos, 1980a; Bonifaz, 1981: 64-67; Matos, 1981b: 195, 200, 204, 214, 216-219, 221-223, 228, 229, 242, 244, 245, 256; Ahuja, 1982; Matos, 1982d: 48; Wagner, 1982; Matos, 1984d, figs. 13-17; González y Olmedo, 1986: 255-263; González, 1987; Matos, 1988: 102, 106; González y Olmedo, s. f.

VI. Glifos.

Glifos en lápidas.

Glifo 4 águila, Contreras y Luna, 1982: 101.

Glifo 1 caña, Matos, 1982d: 70.

Glifo 4 caña, León Portilla, 1981: 40, 44, 66, 69, 70; Matos, 1981b: 168; 1981c: 23; 1984a, foto: 89; Nicholson, 1987: 474; Umberger, 1987: 416, 417; fig. 3; Matos, 1988: 73.

Glifo 3 casa, León Portilla, 1981: 69, 73, 75; Matos, 1981b: 169; 1981c: 37; 1984a, foto: 82, Nicholson, 1987: 474; Umberger, 1987: 418, fig. 5; Matos, 1988: 74, lám. 42.

Glifo 1 conejo, León Portilla, 1981: 69, 70, 73; Matos, 1981b: 168, 169; 1981c: 37; Contreras y Luna, 1982: 82; Matos, 1982d: 33; 1984a, foto: 83; Nicholson, 1987: 474; Umberger, 1987: 417, fig. 4; Matos, 1988: 74, lám. 41.

Glifos 2 conejo y 1 (?) casa, Bonifaz, 1981: 70, 71; Matos, 1981b: 149, 
153; 1981c: 19; 1984a: 86, fotos: 84, 85; Umberger, 1987: 416; Matos, 1988: 70, lám. 36, foto VLLL.

Glifos 13 lluvia y 13 caña labrados en una caja, Matos, 1980a: 78; González, 1982.

Glifos en figurillas: Matos, 1981b: 205, 218, 219; Ahuja, 1982a: 191212; Matos, 1984d, figs. 15-17; Broda, 1987: 230; BCM, 1987: 86.

Glifo en la parte inferior de un caparazón de tortuga en piedra, Matos, 1981b: 318.

VII. Simbolismo del Templo Mayor, León Portilla, 1978; Matos, 1979d; 1980a; 1980b; Carrasco, 1981; Matos, 1981b; 1981c; Zantwijk, 1981; Nicholson, 1982; Matos, 1982a; 1984a: 22-25; 1984d; Nagao, 1985a; 1985b; Matos, 1986d; Broda, 1987; BCM, 1987; Klein, 1987; Kubler, 1987; León Portilla, 1987; Matos, 1987; Pasztory, 1987; Ségota, 1987; Townsend, 1987; Umberger, 1987; Aveni, et al., 1988; Matos, 1988; Broda, s. f.

VIII. Economía, comercio, tributos y el Templo Mayor, Matos, 1979d; 1981b; 1982a; Berdan, 1987; BCM, 1987; Matos, 1988.

IX. Historiografía de las investigaciones sobre el Templo Mayor, Matos, 1981b; 1981c; Valverde, 1982; Matos, 1984a: 40, 41; 1986c: 87 94; Baquedano, 1987: 85-88; Boone, 1987b; Matos, 1988.

\section{BIBLIOGRAFIA}

Aguilera, Carmen

1978a. Coyolxauhqui: Ensayo iconográfico, Cuadernos de la Biblioteca, INAH, México.

1978b. Significado de los rasgos y atavíos de Coyolxauhqui, Boletín del INAH, época III, núm. 24. pp. 81-92, INAH, México.

1979. Coyolxauhqui y la Vía Láctea, Ciencia y Desarrollo, núm. 24, pp. 6-10, México.

1980a. Fechamiento del monolito con el relieve de Coyolxauhqui, Notas de la Biblioteca Nacional de Antropología e Historia, México.

1980b. Reconstrucción de la policromía de Coyolxauhqui, Homenaje al doctor lorge Gurria, UNAM, México.

s.f. Iztac Mixcóatl en la vasija del Templo Mayor, Memoria del Primer Coloquio de Historia de la Religión en Mesoamérica y Áreas Afines, Instituto de Investigaciones Antropológicas, UNAM, México (en prensa). 
Ahuja O., Guillermo

1982a. Excavación de la Cámara II, El Templo Mayor: Excavaciones y estudios, pp. 191-212, INAH, México.

1982b. La cerámica prehispánica en el Templo Mayor, El Templo Mayor: Excavaciones y estudios, pp. 245-252, INAH, México.

- _ - , Alfonso Cruz y Ricardo Sánchez

s.f. Avances y perspectivas de los estudios físico-químicos de algunas piezas del Templo Mayor de Tenochtitlan para publicarse en la Revista Mexicana de Estudios Antropológicos, México.

Álvarez, Ticul

1982. Restos de vertebrados terrestres en la ofrenda 7 y conclusiones, $E l$ Templo Mayor: Excavaciones y estudios, pp. 161-172, INAH, México.

- - - et al.

1982. Relación del material identificado de la ofrenda 7, El Templo Mayor: Excavaciones y estudios, pp. 173-184, INAH, México.

Aveni, Anthony y Horst Hartung

s.f. On the Orientation of the Templo Mayor de Tenochtitlan, Paper presented at the 44th Congress of Americanists, Manchester, England (1982), Mimeografiado.

Aveni, A. F, et al.

1988. Myth, Environment, and the Orientation of the Templo Mayor of Tenochtitlan, American Antiquity, 53 (2), pp. 287-309, Society for American Archaeology.

Baquedano, Elizabeth

1987. Los Aztecas, Editorial Panorama, México.

Berdan, Frances

1987. The Economics of Aztec Luxury Trade and Tribute, The Aztec Templo Mayor, pp. 161-184, Dumbarton Oaks, Washington, D.C.

Blanco Padilla, Alicia

1978. Análisis de los materiales biológicos en las ofrendas a Coyolxauhqui, Boletín del INAH, época 3, núm. 24, pp. 31-38, México.

Bonifaz Nuño, Rubén

1981. El Arte en el Templo Mayor, INAH-SEP, México.

Boone, Elizabeth $\mathrm{H}$.

1987. Templo Mayor Research, 1521-1978, The Aztec Templo Mayor, pp. 5-70, Dumbarton Oaks, Washington, D.C.

Boone, Elizabeth $\mathrm{H}$. (editora)

1987. The Aztec Templo Mayor. A Symposium at Dumbarton Oaks, october 1983, Washington, D.C. 
Broda, Johanna

1982. Der Haupttempel von Mexico-Tenochtitlan, Mexicon, vol. III, no. 6 , enero 1982, pp. 100-102, Berlín.

1987. The Provenience of the Offerings: Tribute and Cosmovision, The Aztec Templo Mayor, pp. 211-256, Dumbarton Oaks, Washington, D.C.

s.f. Ideology of the Aztec State and Human Sacrifice. Conferencia presentada en el simposio Center and Periphery: The Templo Mayor and the Aztec Empire, Societies in Transition (en prensa).

\section{- - - David Carrasco y Eduardo Matos}

1987. The Great Temple of Tenochtitlan. Center and Periphery in the Aztec World, University of California Press, Berkeley.

Cabrera Castro, Rubén

1979. Restos Arquitectónicos del Recinto Sagrado en Excavaciones del Metro y de la Recimentación de la Catedral y Sagrario, El Recinto Sagrado de Méxica-Tenochtitlan, pp. 55-66, INAH, México.

Carrasco, David

1981. Templo Mayor: The Aztec Vision of Place, Journal of Religion, 11: 257-297, Londres.

Catálogos

1980. El Arte del Templo Mayor, Catálogo de la Exposición en el Museo del Palacio de Bellas Artes, México.

1981. Mexique D'hier et D'aujourd'hui, Catálogo de la Exposición en el Musée du Petit Palais, París.

1982. El Templo Mayor de México, Catálogo de la exhibición en el Museo Arqueológico Nacional, Madrid.

1986. Glanz und Untergang des Alten Mexiko. Die Azteken und ihre Vorläufer, 2 vol., Verlag Phillipp von Zabern, Mainz.

Contreras, Eduardo

1979a. La presencia de Tláloc en el Templo Mayor, Ciencia y Desarrollo, núm. 24, pp. 27-29, México.

1979b. Una ofrenda en los restos del Templo Mayor de Tenochtitlan, Trabajos arqueológicos en el centro de la Ciudad de México, pp. 199. 204, INAH, México.

\section{- - - - y Pilar Luna}

1982. Excavaciones, Sección 2, El Templo Mayor: Excávaciones y estudios, pp. 71-102, INAH, México.

Díaz-Pardo, Edmundo

1982. Restos de peces procedentes de la ofrenda 7, El Templo Mayor: Excavaciones y estudios, pp. 151-160, INAH, México.

El Templo Mayor

1981. El Templo Mayor, Bancomer, México, ver López Portillo, José, et. al. 


\section{E1 Templo Mayor}

1982. El Templo Mayor: Excavaciones y estudios, ver Matos Moctezuma, Eduardo (coordinador).

El Templo Mayor

1983. El Templo Mayor, Nonotza, Revista de Difusión Cientifica editada por IBM de México, Año VIII/Cuarto Trimestre, México.

Estrada Balmori, Elma

1979. Ofrendas del Templo Mayor de México-Tenochtitlan, Trabajos arqueológicos en el centro de la Ciudad de México, pp. 183-189, INAH, México.

Franco, María Luisa

1982. El tratamiento de conservación en piedra: tres casos, El Templo Mayor: Excavaciones y estudios, pp. 313-348, INAH, México.

1986. Conservation at the Templo Mayor de Tenochtitlan, In Situ, Archaeological Conservation, pp. 166-175, INAH, GCI, California.

s.f. Conservación del Templo Mayor de Tenochtitlan: Bienes Inmuebles, Tesis de Licenciatura, Escuela Nacional de Conservación, Restauración y Museografía, INAH-SEP, 1986 (en prensa).

Garcia-Bárcena, Joaquin

1979. Fechamiento por Hidratación de Obsidiana de las Excavaciones de la Catedral, El Recinto Sagrado de México-Tenochtitlan, Apéndice 2, pp. 27-28, INAH, México.

García Cook, Angel

1978. Rescate arqueológico del monolito circular de Coyolxauhqui, Boletín del INAH, época III, núm. 24, pp. 18-30, INAH, México.

\section{__ _ - y Raúl M. Arana}

1978. Rescate arqueológico del monolito Coyolxauhqui; informe prelimi. nar, INAH, México.

Gómez Mont, Mercedes

1982. Restos de madera en el adoratorio de Tláloc, El Templo Mayor: Excavaciones y estudios, pp. 233-240, INAH, México.

González Blanco, Salomón

1984. Templo Mayor de México, Trazo i, México.

González, Carlos

1982. La Ofrenda 41, Informe Preliminar, El Templo Mayor: Excavaciones y estudios, pp. 213-220, INAH, México.

1987. Mezcala Style Anthropomorphic Artifacts in the Templo Mayor, Aztec Templo Mayor, pp. 145-160, Dumbarton Oaks, Washington, D.C. 


\section{_ _ _ - y Bertina Olmedo}

1986. Piezas Antropomorfas de Estilo Mezcala, Arqueologia y Etno-histo. ria del Estado de Guerrero, pp. 255-263, INAH, México.

s.f. Presencia del estilo Mezcala en el Templo Mayor; una clasificación de piezas antropomorfas. Tesis, ENAH, INAH, SEP, México, 1986 (en prensa).

Graulich, Michel

1983. Templo Mayor, Coyolxauhqui und Cacaxtla, Mexicon, vol. 5, núm. 5, p. 99 , Berlin.

Gussinyer, Jordi

1979. La Arquitectura Prehispánica en los Alrededores de la Catedral, El Recinto Sagrado de México-Tenochtitlan, pp. 67-74, INAH, México.

Gutiérrez, Isabel

1982. Excavación en la zona norte, El Templo Mayor: Excavaciones y estudios, pp. 103-106, INAH, México.

Gutiérrez Solana, Nelly

1987. Las Serpientes en el Arte Mexica, Instituto de Investigaciones Estéticas, UNAM, México.

s.f. Reseña del libro The Aztec Templo Mayor (publicado por Dumbarton Oaks), Anales del Instituto de Investigaciones Estéticas, núm. 59, UNAM, México (en prensa).

Hasbach, Bảrbara

1982a. Restauración de dos ollas, El Templo Mayor: Excavaciones y estudios, pp. 369.376, INAH, México.

1982b. Restauración de 33 cuchillos ceremoniales policromados, El Templo Mayor: Excavaciones y estudios, pp. 357-368, INAH, Mérico.

Hernández Pons, Elsa

1982a. Excavaciones en el exconvento de Santa Teresa La Antigua, El Templo Mayor: Excavaciones y estudios, pp. 283-294, INAH, México.

1982b. Sobre un conjunto de esculturas asociadas a las escalinatas del Templo Mayor, El Templo Mayor: Excavaciones y estudios, pp. 221232, INAH, México.

Heyden, Doris

1984. Las anteojeras serpentinas de Tláloc, Estudios de Cultura Náhuatl, vol. 17, pp. 23-32, UNAM, México.

1987. Symbolism of Ceramics from the Templo Mayor, The Aztec Templo Mayor, pp. 109.130, Dumbarton Oaks, Washington, D.C.

- - - - y L.F. Villaseñor

1984. The Great Temple and the Aztec Gods. Editorial Minutiae Mexicana, México, 


\section{Huerta Carrillo, Alejandro}

1979. Análisis de la Policromía de los Petroglifos de la Estructura A. El Recinto Sagrado de México-Tenochtitlan, Apéndice 3, pp. 87.94, INAH, México.

Klein, Cecelia F.

1987. The Ideology of Autosacrifice at the Templo Mayor, The Aztec Templo Mayor, pp. 293-370, Dumbarton Oaks, Washington, D.C.

Kubler, George

1987. Summary Perspective, The Aztec Templo Mayor, pp. 485-496, Dumbarton Oaks, Washington, D.C.

León-Portilla, Miguel

1978. México-Tenochtitlan: su espacio y tiempos sagrados, INAH, México.

1981. Los testimonios de la historia, El Templo Mayor, pp. 34-101, Bancomer, México.

1987. The Ethnohistorical Record for the Huey Teocalli of Tenochtitlan, The Aztec Templo Mayor, pp. 71-96, Dumbarton Oaks, Washington, D.C.

López Austin, Alfredo

1979. Iconografía mexica. El monolito verde del Templo Mayor, Anales de Antropología, vol. 16, pp. 133-153, UNAM, México.

1987. The Masked God of Fire, The Aztec Templo Mayor, pp. 257-292, Dumbarton Oaks, Washington, D.C.

López Portillo, José, Miguel León-Portilla y Eduardo Matos M.

1981. El Templo Mayor, Bancomer, México.

Lorenzo, José Luis

1979. Sobre el Templo Mayor de México-Tenochtitlan, Ciencia y Desarro. llo, núm. 24, pp. 11-21, México.

Luna, Pilar

1982. El caracol marino de piedra rosa, El Templo Mayor: Excavaciones $y$ estudios, pp. 240-244, INAH, México.

Mateos Higuera, Salvador

1979. Herencia arqueológica de México-Tenochtitlan, Trabajos arqueológicos en el centro de la ciudad de México, pp. 205-273, INAH, México.

\section{Matos Moctezuma, Eduardo}

1978. El Proyecto Templo Mayor, Boletín del INAH, época III, núm. 24, pp. 3-17, INAH, México.

1979a. El Proyecto Templo Mayor, Ciencia y Desarrollo, núm. 24, pp. 30-33, México.

1979b. Trabajos arqueológicos en el centro de la ciudad de México (coordinador Eduardo Matos Moctezuma), INAH, México. 
1979c. Una Máscara Olmeca en el Templo Mayor de Tenochtitlan, Anales de Antropología, vol. XVI, pp. 11-19, UNAM, México. Publicado también en El Templo Mayor: Excavaciones y estudios, pp. 185-190, INAH, México.

1979d. Imperial Offerings: Tribute and Symbol at the Templo Mayor. Mesa Redonda Center and Periphery, Boulder, Colorado, 1979. Publicado en México, Suplemento cultural del periódico Uno más Uno, 22 de diciembre de 1979.

1980a. El Arte del Templo Mayor, Catálogo de la Exposición en el Museo del Palacio de Bellas Artes, SEP, INAH, México.

1980b. The Great Temple, National Geographic Magazine, vol. 158, núm. 6, pp. 767-775, National Geographic Society, Washington, D.C.

1980c. El Templo Mayor de Tenochtitlan, Antropología Americana, núm. 21, pp. 177-189, Instituto Panamericano de Geografía e Historia, México.

1981a. El Templo Mayor de México: Crónicas del Siglo XVI, Asociación Nacional de Libreros, México.

1981b. Los hallazgos de la arqueología, El Templo Mayor, pp. 103-284, Bancomer, México.

1981c. Una visita al Templo Mayor de Tenochtitlan, INAH, México.

1982a. El Templo Mayor: Economía e Ideología, El Templo Mayor: Excavaciones y estudios, pp. 109-118, INAH, México.

1982b. El Templo Mayor: Excavaciones y estudios (coordinador Eduardo Matos Moctezuma), INAH, México.

1982c. El Templo Mayor: Planos, cortes y perspectivas (coordinador Eduardo Matos Moctezuma, dibujos Víctor Rangel), INAH, México.

1982d. Las Excavaciones del Proyecto Templo Mayor (1978-1981) y Excavaciones en la Sección I, El Templo Mayor: Excavaciones y estudios, pp. 11-70, INAH, México.

1983. Notas sobre algunas urnas funerarias del Templo Mayor, Jahrbuch fïr Geschichte... 20, pp. 17-32, Böhlau Verlag Köln.

1984a. Guia oficial. Templo Mayor, INAH-Salvat, México.

1984b. Los edificios aledaños al Templo Mayor, Estudios de Cultura Náhuatl, vol. 17, pp. 15-21, UNAM, México.

1984c. The Great Temple of Tenochtitlan, Scientific American, vol. 251, núm. 2, pp. 80-89, Nueva York.

1984d. The Templo Mayor of Tenochtitlan: Economics and Ideology, Ritual Human Sacrifice in Mesoamerica, pp. 133-164, Dumbarton Oaks, Washington, D.C.

1986a. Der Templo Mayor, Glanz und Untergang des Alten Mexiko, pp. 105-120, Verlag Philipp von Zabern, Mainz.

1986b. El Proyecto Templo Mayor, Arqueología y Etnohistoria del Estado de Guerrero, pp. 249-252, INAH, México.

1986c. Los dioses que se negaron a morir. Arqueologia y crónicas del Templo Mayor, prólogo, introducción, selección y notas de Eduardo Matos Moctezuma, SEP, México. 
1986d. Vida y muerte en el Templo Mayor, Ed. Oceano, México.

1987. Symbolism of the Templo Mayor, The Aztec Templo Mayor, pp. 185-210, Dumbarton Oaks, Washington, D.C.

1988. The Great Temple of the Aztecs. Treasures of Tenochtitlan, Thames and Hudson, Londres.

s.f. El Templo Mayor: Excavaciones y estudios, vol. 2 (coordinador Eduardo Matos Moctezuma), INAH, México (en prensa).

- - - - y Felipe Ehremberg

1980. Coyolxauhqui, SEP, México.

- - - - y Guillermo Ahuja

s.f. Guía del Museo del Templo Mayor (en prensa).

Mẻndez G. Miguel y Joaquín García-Bárcena

1979. El Instrumental Lítico: Clasificación y Cronología, El Recinto Sagrado de México-Tenochtitlan, pp. 29-36, INAH, México.

Mercado, Vida

1982. Restauración de dos urnas, El Templo Mayor: Excavaciones y estudios, pp. 349-356, INAH, México.

Molina, Augusto

1980. The Building of Tenochtitlan, National Geographic Magazine, vol. 158, núm. 6, pp. 753.764, National Geographic Society, Washington, D.C.

1987. Templo Mayor Architecture: So What's New, The Aztec Templo Mayor, pp. 97-108, Dumbarton Oaks, Washington, D.C.

Monjarás-Ruiz, Jesús

1984. Reseña bibliográfica de El Templo Mayor de México de López Portillo, Y., M. León-Portilla y E. Matos, Estudios de Cultura Náhuatl, núm. 17, pp. 369-370, UNAM, México.

Nagao, Debra

1985a. Mexica Buried Offerings: a Historical and Contextual Analysis, Bar International Series 235, Oxford.

1985b. The Planting of Sustenance: the Symbolism of the Two-Horned God in Mexica Offerings from the Templo Mayor, Res-Anthropology and Aesthetics, no. 10, pp. 5-27, Peabody Museum, Harvard University.

s.f. Recovering the Mind of the Aztec State; a Contextual Analysis of Artifacts as Ritual, Manuscript 1984, Department of Art History and Archaelogy, Colombia University, Nueva York.

Nicholson, Henry B.

1982. Revelation of the Great Temple, Natural History, vol. 91, núm. 7, pp. 48-58, American Museum of Natural History, Nueva York. 
1985. The New Tenochtitlan Templo Mayor Coyolxauqui-Chantico Monument, Indiana, vol. 10, part 2, pp. 77-98, Berlín.

1987. Symposium on the Aztec Templo Mayor: Discussion, The Aztec Templo Mayor, pp. 463-484, Dumbarton Oaks, Washington, D.C.

- - - y Eloise Quiñones

1983. Art of Aztec Mexico, Treasures of Tenochtitlan, National Gallery of Art, Washington, D.C.

Noguez, Xavier

1988. Reseña de The Great Temple of Tenochtitlan: Center and Periphery in the Aztec World por J. Broda, D. Carrasco y E. Matos M., Hemisphere, vol. 1, núm. 1, pp. 39-40, Florida International University, Miami.

Pasztory, Esther

1983. Aztec Art, Harry N. Abrams, Nueva York.

1987. Texts, Archaeology, Art, and History in the Templo Mayor: Reflections, The Aztec Templo Mayor, pp. 451-462, Dumbarton Oaks, Washington, D.C.

Peña Gómez, Rosa María

1978. Análisis de los restos humanos en las ofrendas a Coyolxauhqui, Boletín del INAH, época III, núm. 24, pp. 39-51, INAH, México.

Polaco, Oscar

1982. Los invertebrados de la ofrenda 7 del Templo Mayor, El Templo Mayor: Excavaciones y estudios, pp. 143-150, INAH, México.

1986. Restos biológicos de la Costa del Pacífico, Arqueología y Ethnohistoria del Estado de Guerrero, pp. 267-275, INAH, México.

Reyes Cortés, Manuel.

1978. Estratigrafía del área Templo Mayor-Catedral Metropolitana, Boletín del INAH, época III, núm. 24, pp. 52-71, INAH, México.

1979. Apéndice I: Estratigrafía de Dos Pozos Realizados en el Jardín de la Catedral, El Recinto Sagrado de México-Tenochtitlan, pp. 21-26, INAH, México.

\section{- - - y Joaquín García-Bárcena}

1979. Estratificación en el área de la Catedral, El Recinto Sagrado de México-Tenochtitlan, pp. 17-20, INAH, México.

Riese, Berthold

1982. Coyolxauhqui, Miscelánea Ibero-Americana, Berlín.

Román, Juan Alberto

1987. Offering 48 of the Templo Mayor: A Case of Child Sacrifice, The Aztec Templo Mayor, pp. 131-144, Dumbarton Oaks, Washington, D.C.

s.f. El sacrificio de niños en honor a Tláloc. La ofrenda 48 del Templo 
Mayor, tesis de licenciatura ENAH, INAH-SEP, México, 1986 (en prensa).

Romero Galván, José Rubén

1979. Notas sobre el Templo Mayor de Tenochtitlan (Descripción y hallazgos arqueológicos), El Recinto Sagrado de México-Tenochtitlan, pp. 9-16, INAH, México.

Santaella, Yolanda

1982. Informe de la sección de restauración, El Templo Mayor: Excavaciones y estudios, pp. 295-312, INAH, México.

Ségota, Durdica

1987. Unidad binaria del Templo Mayor de Tenochtitlan. Hipótesis de trabajo, Anales del Instituto de Investigaciones Estéticas, vol. 58, pp. 47-54, UNAM, México.

Simoni-Abbat, Mireille

1982. Le Templo Mayor de México, Archeo, 162, pp. 6-9.

Teniente Nivón, Edmundo

s.f. Análisis ictiofaunístico de los restos encontrados en el Templo Mayor, tesis de licenciatura, Instituto Politécnico Nacional, México, 1986 (en prensa).

The Aztec Templo Mayor

1987. The Aztec Templo Mayor, A Symposium at Dumbarton Oaks, Dumbarton Oaks, Washington, D.C.

Thiemer-Sachse, Ursula

1980. Hallazgos arqueológicos en el centro de la capital mexicana, Anales de Antropología, vol. XVII, tomo I, pp. 115-128, UNAM, México.

Townsend, Richard F.

1987. Coronation at Tenochtitlan, The Aztec Templo Mayor, pp. 371-410, Dumbarton Oaks, Washington, D.C.

Umberger, Emily

1982. Aztec Mexico: Discovery of the Templo Mayor. Pamphlet published in conjunction with the exhibition at the American Museum of $\mathrm{Na}$ tural History, Nueva York.

1987. Events Commemorated by Date Plaques at the Templo Mayor: Further Thoughts on the Solar Metaphor, The Aztec Templo Mayor, pp. 411-459, Dumbarton Oaks, Washington, D.C.

Valyerde, Adrián

1982. Algunos hallazgos arqueológicos en el centro de la Ciudad de México, Anales de Antropologia, vol. XIX, pp. 45-49, UNAM, México.

Vega Sosa, Constanza

1978. Datos para una cronología relativa en el área del recinto sagrado de 
México-Tenochtitlan, Boletín del INAH, época 3, núm. 24, pp. 72-80, INAH, México.

1979a. El Recinto Sagrado de México-Tenochtitlan. Excavaciones 1968-69 1975-76 (coordinadora Constanza Vega Sosa), INAH, México.

1979b. El Templo del Sol. Su Relación con el Glifo Chalchihuitl. El Templo de Ehécatl-Quetzalcóatl, El Recinto Sagrado de México-Tenochtitlan, pp. 75-86, INAH, México.

- - - - y Manuel Reyes Cortés

1979. La Cerámica: Clasificación y Cronología, Análisis Petrográfico, Recinto Sagrado de México-Tenochtitlan, pp. 37-54, INAH, México.

Villalobos Pérez, Alejandro

1985. Consideraciones sobre un plano reconstructivo del recinto sagrado de México-Tenochtitlan, Cuadernos de Arquitectura Mesoamericana, núm. 4, pp. 57-64, UNAM, México.

Wagner, Diana

1982. Reporte de las ofrendas excavadas en 1978, El Templo Mayor: Excavaciones y estudios, pp. 119-142, INAH, México.

Winning, Hasso von

1985. Ein Kommentar zu Michel Graulich: Templo Mayor, Coyolxauhqui und Cacaxtla, Mexicon, vol. VII, núm. 4, pp. 57-60, Berlín.

Zantwijk, Rudolf van

1981. The Great Templo of Tenochtitlan. Model of Aztec Cosmovision, Mesoamerican Sites and Worldviews, pp. 71-86, Dumbarton Oaks, Washington, D.C. 